\title{
Individual Differences in Amygdala Activity Predict Response Speed during Working Memory
}

\author{
Alexandre Schaefer, ${ }^{1}$ Todd S. Braver, ${ }^{2}$ Jeremy R. Reynolds, ${ }^{2}$ Gregory C. Burgess, ${ }^{2}$ Tal Yarkoni, ${ }^{2}$ and Jeremy R. Gray ${ }^{1}$ \\ ${ }^{1}$ Psychology Department, Yale University, New Haven, Connecticut 06520, and 2Department of Psychology, Washington University, St. Louis, Missouri 63130
}

The human amygdala has classically been viewed as a brain structure primarily related to emotions and dissociated from higher cognition. We report here findings suggesting that the human amygdala also has a role in supporting working memory (WM), a canonical higher cognitive function. In a first functional magnetic resonance imaging (fMRI) study ( $n=53$ ), individual differences in amygdala activity predicted behavioral performance in a 3-back WM task. Specifically, higher event-related amygdala amplitude predicted faster response time (RT; $r=-0.64$ ), with no loss of accuracy. This relationship was not contingent on mood state, task content, or personality variables. In a second fMRI study $(n=21)$, we replicated the key finding $(r=-0.47)$ and further showed that the correlation between the amygdala and faster RT was specific to a high working memory load condition (3-back) compared with a low working memory load condition (1-back). These results support models of amygdala function that can account for its involvement not only in emotion but also higher cognition.

Key words: amygdala; working memory; emotion; cognitive control; goal-relevance; fMRI

\section{Introduction}

The amygdala is unambiguously a core part of the emotional brain (LeDoux, 1996; Morris et al., 1996; Damasio et al., 2000; Vuilleumier et al., 2001; Pessoa et al., 2002a; Zald, 2003; Whalen et al., 2004). The wealth of data supporting the link between the amygdala and emotional functions is such that the human amygdala is often used to illustrate the segregation of emotion and higher cognition within the brain (Phelps, 2005). However, indirect evidence hints that the human amygdala could also be involved in higher cognitive functions such as working memory (WM) and executive control.

It is known from animal research that the amygdala has a general role in attention and vigilance processes beyond its emotional functions (Kapp et al., 1992; Holland and Gallagher, 1999; Davis and Whalen, 2001; Sander et al., 2003; Holland and Gallagher, 2006). Specifically, it has been suggested that one of the functions of the amygdala was to increase vigilance levels to specific categories of stimuli to facilitate the adaptation to goalrelevant situations. (Whalen, 1998; Davis and Whalen, 2001). Regarding more specific links with WM, there is some evidence that the amygdala can contribute to performance in WM tasks in rats (Peinado-Manzano, 1990; McIntyre et al., 2003). In humans, the only evidence for a relationship between WM and the amygdalae is scarce and indirect, consisting of two studies investigating the role of dopamine (DA) in WM. A microdialysis study in

Received June 19, 2006; revised Aug. 15, 2006; accepted Aug. 22, 2006.

This study was supported by grants from National Institutes of Health (MH 66088). We thank Ajay Satpute, Christine Hoyer, and John Zelenski for their contribution in data collection.

Correspondence should be addressed to Jeremy R. Gray, Yale University, Psychology Department, Box 208205, New Haven, CT 06520. E-mail: Jeremy.Gray@yale.edu.

A. Schaefer's present address: University of Leeds, Leeds LS2 9JT, UK.

J. R. Reynolds' and B. C. Burgess' present address: University of Colorado at Boulder, Boulder, C0 80309.

DOl:10.1523/JNEUROSCI.2567-06.2006

Copyright $\odot 2006$ Society for Neuroscience ～0270-6474/06/2610120-09\$15.00/0 human epileptic patients found that extracellular levels of DA increased in the amygdala during the performance of a WM task compared with a reading task (Fried et al., 2001). Importantly, the task did not have any overt emotional component that differentiated it from the control task. This result is consistent with a positron emission tomography study on human subjects showing an increased DA release in medial temporal areas during a WM task compared with a letter discrimination task (Aalto et al., 2005). However, these studies did not show a relationship between amygdala activity and WM behavioral performance, which allows the interpretation that DA release in the amygdala might have been caused by heightened levels of stress during a difficult WM task compared with an easier control task.

We report here two functional magnetic resonance imaging (fMRI) studies that, to our knowledge, are the first to show a significant relationship between individual differences in human amygdala activity and WM performance. In both experiments, subjects were scanned as they performed variations of the $n$-back working memory task. In the first study, we observed a strong relationship between individual differences in event-related amygdala activity and 3-back response time (RT). We followed up this finding with a number of post hoc analyses showing that the results held even when eliminating or controlling for a number of variables that could potentially provide an affective source for the amygdala-performance relationship. In the second study, we first sought to replicate the key result. We also manipulated WM load to assess whether amygdala activity was related to performance under a high working memory load condition specifically.

\section{Materials and Methods \\ Experiment 1}

The primary goal of experiment 1 was to identify brain regions that showed significant correlations with individual differences in 3-back task 


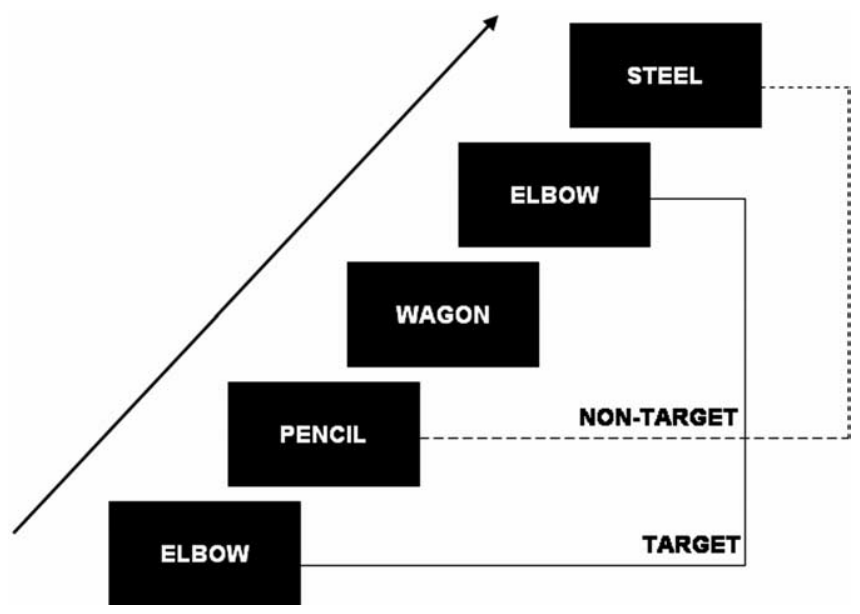

Figure 1. Schematic diagram of an $n$-back task. Stimuli are displayed sequentially on a screen and subjects are instructed to decide through a button press whether the current stimulus is equal or different from the stimulus displayed $n$ trials back. In this example, $n=3$.

performance (response speed) using a whole-brain voxelwise correlational analysis. We observed a strong relationship in the amygdala, which prompted a more focused set of follow-up analyses. In these follow-up analyses, we used an anatomical region of interest (ROI) approach to determine whether the relationship between the amygdala and behavioral performance was influenced by mood, task content, or personality differences. We used an individual-differences approach in part to provide a fine-grained measure of brain-behavior relationships and in part to allow us to control statistically for individual differences in affective responses and dispositions (Kosslyn et al., 2002).

\section{Subjects}

Participants were healthy, right-handed, native English speakers $(n=60$; 29 males; age range, 18-37 years) from Washington University and the surrounding community screened for no history of neurological disorder, current psychoactive medication, or factors contraindicating fMRI. All participants gave written informed consent. The experiment was approved by the Washington University Medical Center Human Subjects Committee. Seven participants had fMRI datasets that were compromised by excessive head movement, technical problems, or too few trials for estimating event-related responses, leaving $n=53$. Analyses of this dataset have been published previously, addressing different questions, with none focused on the amygdala (Gray et al., 2003, 2005).

\section{Experimental tasks}

The 3-back task was administered using PsyScope (Cohen et al., 1993) on a Macintosh G3 (Apple Computer, Cupertino, CA). Participants performed a standard version of a task that has been used in many previous studies of working memory, the 3-back task (Fig. 1). Stimuli (either words or faces) were displayed sequentially on a screen. Participants were instructed to respond with their right index finger if the current stimulus matched the stimulus presented three trials back (target response), and with their right middle finger if the current stimulus was different (nontarget response). For each subject, performance was assessed as the median reaction time (RT) of correct responses. For a given scanning run, stimuli were either all faces (unfamiliar, attractive males and females intermixed, either neutral or smiling) or all words (concrete English nouns with neutral content, e.g., rooster, elbow, steel, fence, ball, tooth). These stimuli were used in a previous study (Gray et al., 2002). Stimuli were shown one per trial for $2.0 \mathrm{~s}$, with a fixation point (cross-hair) shown between stimuli. Each scanning run had four unanalyzed trials, followed by four blocks of 21 task trials ( 16 task stimuli with five crosshair fixation trials randomly interspersed to introduce temporal jitter) and $23.6 \mathrm{~s}$ (10 trials) of resting fixation (a dash), for a total of 128 trials per scanning run ( $2.36 \mathrm{~s}$ per trial). Just before each scanning run, participants watched one of six 7 min videos, two of which were emotionally neutral and four of which were emotionally evocative (order counterbalanced).
The emotional films were either positively or negatively valenced (Gray, 2001, 2002). After each scanning run, subjects were asked to rate how they felt using the five-point Profile of Mood States (POMS) scales (McNair et al., 1971) for each of the following adjectives: amused, calm, fatigued, gloomy, energetic, and anxious. Before the scanning sessions, participants were asked to fill in a series of questionnaires and tests, including the behavioral inhibition system-behavioral activation system (BIS-BAS) scales (Carver and White, 1994) and the revised Eysenck Personality Questionnaire (Eysenck and Eysenck, 1975). In addition, participants completed the Raven's Advanced Progressive Matrices (Raven et al., 1998), following the method described in a previous article (Gray et al., 2003).

\section{fMRI scanning}

Whole-brain images were acquired on a 1.5 Tesla Vision System (Siemens, Erlangen, Germany). Structural images were acquired using an MP-RAGE (magnetization-prepared rapid-acquisition gradient-echo) T1-weighted sequence. Functional images were acquired using an asymmetric spin-echo echo-planar sequence sensitive to blood oxygen leveldependent (BOLD) magnetic susceptibility [repetition time (TR), 2360 $\mathrm{ms}$; echo time (TE), $50 \mathrm{~ms}$; flip angle, $90^{\circ}$. Each scanning run gave 128 sets of brain volumes ( 16 contiguous, 8 -mm-thick axial images, $3.75 \times$ $3.75 \mathrm{~mm}$ in-plane resolution). After movement and artifact correction, functional images were normalized within each scanning run and temporally aligned within each brain volume. Functional images were resampled into $3 \mathrm{~mm}$ isotropic voxels, transformed into atlas space (Talairach and Tournoux, 1988) and smoothed with a Gaussian filter $(9 \mathrm{~mm}$ full-width half-maximum) before statistical analysis. Although a $9 \mathrm{~mm}$ filter can be considered adequate to detect amygdalar activations (Zald, 2003), we also analyzed and reported results obtained without spatial smoothing, to ensure that active voxels were localized in the amygdala proper within a $3 \mathrm{~mm}$ range.

\section{Data analysis}

A general linear model (GLM) approach (Friston et al., 1995) was used to estimate event-related and sustained neural activity (Visscher et al., 2003). Event-related effects were analyzed by estimating values for the time points within the hemodynamic response epoch, taken to have a $17 \mathrm{~s}$ duration (7 scan frames). Additionally, sustained regressors coded the task blocks relative to blocks of fixation (Visscher et al., 2003). Two nuisance variables for each BOLD run were also included: a baseline and linear trend.

Correlational analysis: amygdala and task performance. We first conducted a whole-brain voxelwise search for voxels whose amplitude was negatively correlated with RT, with all experimental conditions collapsed in a single 3-back condition (i.e., activity was estimated ignoring distinctions among mood and stimulus conditions, all trials were considered to belong to a generic 3-back task condition). The amplitude of eventrelated activity was defined as the difference between the peak and the onset of the hemodynamic response, calculated for all voxels, each condition and each subject, based on psychophysiological signal analysis principles (Stern et al., 2001). First, a voxel-level threshold of $p<0.0001$ (uncorrected) combined with a cluster-size threshold of at least nine voxels (which results in a cluster-level $p<0.05$ threshold) (McAvoy et al., 2001) was applied. Next, we conducted the same analysis with a threshold of $p<0.001$ (uncorrected) for exploratory purposes. To address potential localization issues caused by spatial smoothing, a complementary analysis was performed without spatial smoothing, in which we looked for voxels correlated with RT within the region of the amygdala proper (defined below) using a threshold of $p<0.001$ (uncorrected). We also performed analyses looking for correlations between the amygdala and accuracy measures ( $d^{\prime}$ and false alarm rate). We found significant results in experiment 1 showing that increased amygdala activity was related to a lower false alarm rate and a higher $d^{\prime}$, thus consistent with the results obtained with RT. However, as these results were not replicated in experiment 2, we decided not to consider them further. The absence of speedaccuracy trade-off and significant correlations between RT and accuracy (see Results) ascertained that RT could be considered as a reliable measure of task performance. 
Table 1. Regions correlated with 3-back reaction time

\begin{tabular}{lrrrrr}
\hline & \multicolumn{7}{l}{ Talairach coordinates } \\
\cline { 2 - 6 } Area & $x$ & $y$ & \multicolumn{1}{l}{$z$} & \multicolumn{1}{l}{ r } & \multicolumn{1}{c}{ Z } \\
\hline Left amygdala & -20 & -6 & -21 & -0.64 & -5.1 \\
Right amygdala & 22 & -3 & -21 & -0.61 & -4.8 \\
Middle frontal (BA 6/8) & -38 & 0 & 57 & -0.51 & -3.9 \\
Insula (BA 13) & -44 & -3 & 3 & -0.50 & -3.8 \\
Superior temporal gyrus (BA 38) & -34 & 6 & -24 & -0.45 & -3.4 \\
Cerebellum & -26 & -90 & -36 & -0.48 & -3.6 \\
\hline
\end{tabular}

$p<0.001$ (uncorrected) for all regions. Reported coordinates are peaks.

Hierarchical regression analyses. Two sets of hierarchical regression analyses were conducted to examine the anatomical specificity of the results obtained through the voxelwise analysis. First, we examined whether the amygdala-RT relationships observed through the voxelwise analysis were not attributable to a global whole-brain correlation with RT. Specifically, we selected all the amygdala voxels that were revealed to be correlated with RT in the voxelwise analysis using a threshold of $p<$ 0.001 and created a region of interest (separately for left and right amygdala). Next, we selected all the voxels of the brain minus the amygdala voxels and created a whole-brain volume not including the amygdala (but including all the other regions correlated with RT) (Table 1). Next, we extracted amplitude values for the amygdala ROI and the whole-brain volume. Amplitude values of the whole-brain volume were then entered as a single predictor in a regression model with RT as the dependent measure. Next, we entered one of the amygdala ROIs (left or right) as a second predictor into the regression model. We then tested the significance of the $\Delta R^{2}$ associated to the addition of the amygdala to the model using a statistical threshold of $p<0.05$. The second hierarchical regression analysis examined whether the amygdala had a significant unique contribution to RT relative to other regions predicting task performance. We identified a network of performance-related regions by clustering together all the voxels predicting RT at $p<0.001$ (Table 1), except amygdala voxels. Amplitude values of the voxels belonging to the performance-related network were averaged and entered as a single predictor in a regression model with RT as the dependent measure. Next, we entered one of the amygdala ROIs (left or right) as a second predictor into the regression model, and tested the significance of the $\Delta R^{2}$.

Follow-up ROI analyses. A follow-up set of ROI analyses were also conducted to test whether the original results could be confirmed (1) on the basis of strict anatomical criteria for amygdala definition, and (2) after controlling for the possible role of affect-related variables. ROIs were created for both the left and right amygdalae on the basis of strict a priori anatomical criteria [i.e., the limits of the proper amygdala as defined in Talairach and Tournoux's atlas (1988)]. The ROIs extended from 17 to $30 \mathrm{~mm}$ lateral to the midline $(x), 1 \mathrm{~mm}$ anterior to 11 posterior to the anterior commissure $(y)$, and 7-21 mm below the intercommissural line $(z)$ (Zald, 2003). For both left and right amygdala ROIs, the time course of the event-related response of all the voxels in the ROI were averaged in a single ROI time course for each experimental condition and for each subject. For each subject, the amplitude of amygdala activity was estimated by subtracting the onset from the peak of the time course, consistent with the principles mentioned above. Pearson correlations were computed between these amygdala activation values and performance scores, using a statistical threshold of $p<0.05$ (two-tailed). Correlations were computed separately for each emotional mood category and for each stimulus content (faces vs words). In addition, pairwise comparisons of the correlation coefficients were performed using the Pearson-Filon modified $Z(Z P F)$ statistic (Raghunathan et al., 1996) (threshold: $Z P F= \pm 1.65 ; p<0.05$ ).

Furthermore, we ran multiple regression analyses to verify whether the effects of amygdala on RT could be explained by confounding emotional and personality variables. We first tested a series of simple regression models in which the amplitude values of either the left or right amygdala ROIs for 3-back task activity were entered alone as regressors and RT as a dependent variable. Regression coefficients were significant for both left and right ROIs $(\beta=-0.48, p<.001$ and $\beta=-0.56, p<.001$, respec- tively). Next, the regression was retested entering amygdala values in the model simultaneously with one of the potential confounding variables (the POMS scales, the extraversion and neuroticism scales of the Eysenck Personality Questionnaire, and the BIS-BAS scales). Next, $t$ tests compared the simple regression coefficients of the effects of amygdala on RT (when the amygdala is the only regressor in the equation) with the partial coefficients (when a confounding variable is added to the model) to see whether controlling for potential confounds had induced significant changes in the magnitude of the effects of amygdala activity on performance. Further, we also tested correlations between the amygdala and Ravens matrices to examine whether amygdala activity was affected by individual differences in fluid intelligence.

\section{Experiment 2}

The primary goal of experiment 2 was to replicate the relationship between the amygdala and improved behavioral performance in a 3-back task using only neutral words and no mood induction. The second experiment was, thus, very similar to the "neutral mood-only words" condition of experiment 1 . A secondary goal was to examine whether the amygdala-RT relationship could be moderated by working memory load. The brain activity of 21 participants was then assessed with fMRI, in which they had to perform two versions of the $n$-back task: a 1-back (low cognitive load) and a 3-back task (high cognitive load).

We predicted that we would replicate the findings of experiment 1 (i.e., a relationship between amygdala activity and improved performance in the 3-back condition). Further, we hypothesized that if the amygdala is specifically involved in WM performance rather than general cognitive performance, correlations with a faster response speed should be specific to the high-load condition. Finally, we hypothesized that if amygdala is truly involved in WM task performance, then the amplitude of its responses should be higher in a high load condition compared with a low load condition.

\section{Participants}

Twenty-one right-handed participants with no evidence of neurological compromise participated in this study. Participants were 7 males and 14 females with a mean age of 21.5 years (range, 19-29 years). Participants gave informed consent per guidelines set by the Washington University Medical Center Human Studies Committee and were paid 25 dollars for each hour of participation.

\section{Behavioral tasks}

Participants performed a standard version of the $n$-back task having only neutral words. There were two different conditions. The 3-back condition was similar to the 3-back task used in the first study. In the 1-back condition, participants had to decide whether the current word matched the word presented in the immediately previous trial (i.e., one trial back). Thus, the WM load was manipulated from the 1- to 3-back conditions. Participants were instructed to respond with their right index finger to targets and with their right middle finger to nontargets. Stimuli were pseudorandom sequences of neutral words (e.g., shirt, wire, ladder, oven, pupil, street, ring, tube, closet). Each block of trials started with an instructional cue lasting for $5 \mathrm{~s}$ indicating what task to perform. Following this instructional cue, a block of 36 trials began. Each word was on the screen for $2000 \mathrm{~ms}$ and was followed by a variable intertrial interval (500-13000 ms, distributed exponentially) to allow for estimation of the event-related hemodynamic response. Participants performed between one and four blocks at each level of load.

\section{Functional imaging and data analysis}

The scanning and analysis parameters were similar to those of the first study and we describe hereafter parameters that differed. Functional images were acquired using a TR of $2500 \mathrm{~ms}$. Task blocks were $150 \mathrm{~s}$ long, whereas fixation blocks were $37.5 \mathrm{~s}$ in duration. Each run lasted $\sim 7 \mathrm{~min}$ (165 whole-brain acquisitions), and a 2 min delay occurred between runs, during which time participants rested. Each whole-brain volume consisted of 18 contiguous 7 -mm-thick axial images with $3.75 \times 3.75$ $\mathrm{mm}$ in-plane resolution. Preprocessing was identical to that seen in experiment 1 , except that (1) a cubic spline interpolation was used to tem- 


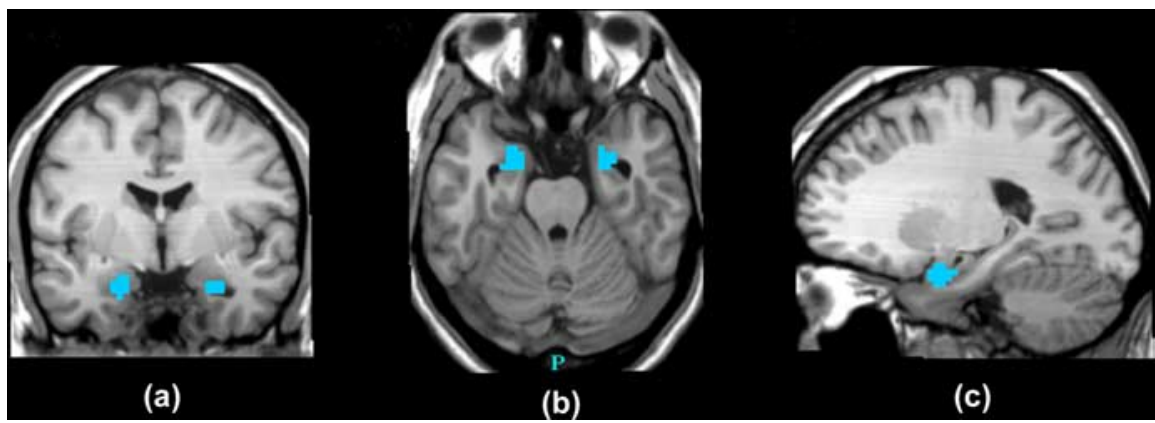

Figure 2. Statistical map of Pearson correlations between brain activity and task response time in experiment 1 (threshold, $p<0.05$, cluster-level corrected) showing clusters of negative correlations in the left and right amygdala on coronal ( $\boldsymbol{a})$, transverse (b), and sagittal (c) views (peak voxel correlation: $x=-20, y=-6, z=-21$ ).

hemodynamic response of each ROI (for each of the 3-back and 1-back conditions) and RT. After an outlier analysis, one subject was dropped out of the correlation analysis because it appeared that he was driving a significant portion of the effects (covariance ratio, 2.4; $z=$ 4.00; original correlation between the left amygdala ROI and RT, $r=-0.54$ ). A hierarchical regression testing the unique contribution of the amygdala to RT controlling for wholebrain activity was performed following the same methods used in experiment 1 . The statistical threshold was $p<0.05$.

We also examined whether the correlation between amygdala activity and RT was moderated by cognitive load by comparing the correlation coefficients between amygdala activity and 3-back RT with the correlation between

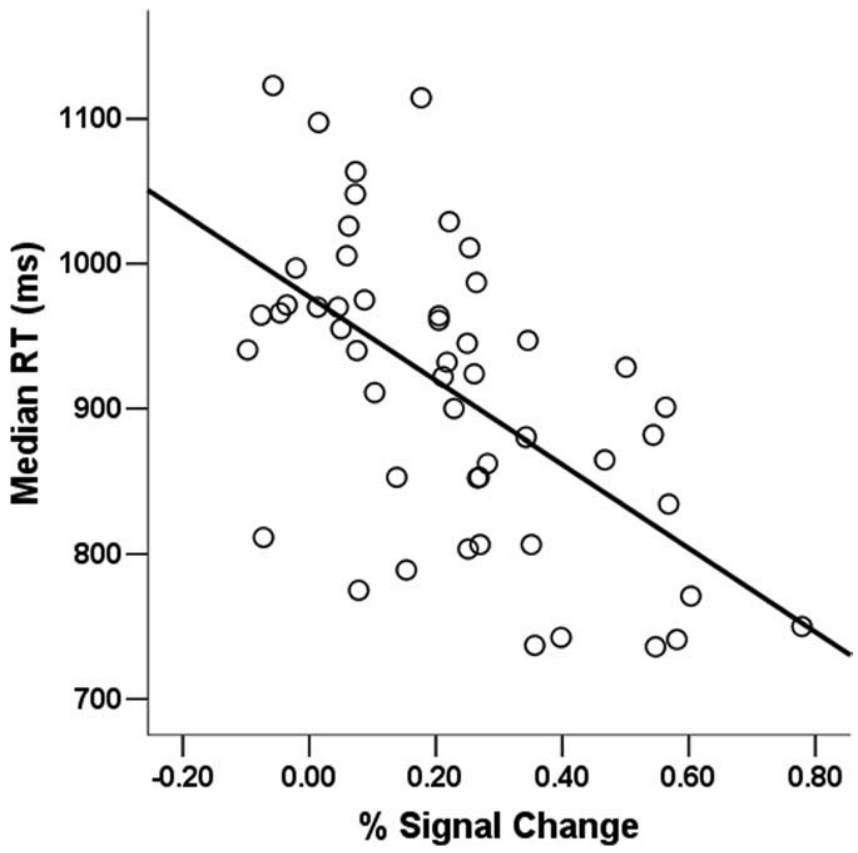

Figure 3. Scatterplot of the peak voxel correlation between the left amygdala and response time in experiment 1 (after controlling for outliers): $x=-20, y=-6, z=-21 ; r=-0.60$; $p=0.000001$.

porally realign scans rather than a sinc function and (2) no smoothing was performed.

The same amygdala anatomical ROIs used in experiment 1 were used in experiment 2. The time course of BOLD data were averaged across all voxels in the anatomically defined left and right amygdala ROIs, resulting in two time-series (one each for the 1-back and 3-back conditions). Similar to experiment 1, a GLM was used to estimate the hemodynamic response for each of these average time courses. Different time points were coded within the hemodynamic response epoch, taken to have a $17.5 \mathrm{~s}$ duration (seven scan frames). Additionally, two sustained regressors (for the 1- and 3-back conditions) coded the task blocks relative to blocks of fixation (Visscher et al., 2003). Similar to experiment 1, two nuisance variables for each BOLD run were included: a baseline and linear trend.

A similar approach in the analysis of event-related responses was taken as in experiment 1 . To assess the amplitude of event-related amygdala activity, the difference between the peak and the onset of the average estimated hemodynamic response was calculated for all voxels, each condition and each subject, based on psychophysiological signal analysis principles (Stern et al., 2001).

Next, a set of correlations was computed between the summarized amygdala activity and 1-back RT using the ZPF statistic. Because a significant moderation effect was found in the left amygdala (see Results), we proceeded to additional analyses to explore this effect. First, we tested the correlation between left amygdala activity in the 3-back and left amygdala activity in the 1-back conditions. Second, we tested the hypothesis that the difference between 3-back and 1-back left amygdala activity would be more important for high performers. We then separated the sample in a group of high performers (H-P) and a group of low performers (L-P). For each subject, a score was created by averaging the standardized RT values of 3-back and 1-back conditions. Next, the two groups were created by computing a median split on this variable. Then, we computed a cognitive load (3-back vs 1-back) by performance (H-P vs L-P) ANOVA, followed by $F$ tests of the load manipulation on amygdala activity separately for H-P and L-P groups. Third, a complementary correlational analysis examined whether the change in amygdala activity from 1-back to 3-back conditions (3-back minus 1-back) could predict RT in both 3-back and 1-back conditions. Outliers of correlation analyses were excluded using the covariance ratio technique described above. In addition, the ANOVA described above was computed on the right amygdala ROI as well. Statistical thresholds were at $p<0.05$.

\section{Results \\ Experiment 1 \\ Behavioral results}

Participants had to perform six blocks of the 3-back version of the $n$-back task. The average of participants' median RT was $915 \mathrm{~ms}$ (SD, 113.3). They had a mean accuracy [estimated with the $d^{\prime}$ signal detection parameter (Snodgrass and Corwin, 1988] of $d^{\prime}=$ $2.24(\mathrm{SD}, 0.54)$, and a mean false alarm rate $(\mathrm{FA})=0.08(\mathrm{SD}$, $0.04)$. In addition, a negative correlation between RT and $d^{\prime}$ was found $(r=-0.29 ; p=0.03)$, as well as a positive correlation between RT and false alarm rate $(r=0.28$; $p=0.04)$, which indicated that there was no speed-accuracy trade-off. In addition, task performance was affected by mood, stimulus content, and personality measures (supplemental Tables 1, 2, available at www. jneurosci.org as supplemental material), consistent with our previous work (Gray et al., 2002, 2005).

\section{Imaging results}

Amygdala and task performance. A whole-brain search for voxels negatively correlated with RT revealed only two significant clusters using a stringent statistical threshold $(p<0.05$ cluster-level corrected). These clusters correspond to the left and right amygdalae (left peak: $x=-20, y=-6, z=-21$; right peak: $x=22$, $y=-3, z=-21)$, indicating that amygdala voxels were the most potent predictors of RT in the 3-back task (Figs. 2, 3). Next, we conducted the same analysis with a threshold of $p<0.001$ (uncorrected) for exploratory purposes, and found six activation clusters that showed significant negative correlations with RT 
(Table 1). To address potential localization issues caused by spatial smoothing, the same analyses were performed without previous smoothing. With a threshold of $p<0.001$ (uncorrected), voxels negatively correlated with task response time were observed both in the left and right amygdalae (left peak: $x=22, y=$ $-6, z=-21$; right peak: $x=26, y=0, z=-21)$. In addition, we performed an outlier analysis to verify whether observed correlations were not driven by single subjects. After exclusion of two outliers (covariance ratios, $1.4,0.81 ; Z$ values $=4.2,-3.0$ ), the peak voxel correlation remained highly significant $(r=-0.60$, $p<0.000001$; original correlation: $r=-0.64, p=0.0000001$ ) (Fig. 3), indicating that the effects were not driven by single individuals.

Hierarchical regression analyses. The unique contribution of the amygdala to the prediction of RT was highly significant while controlling for whole-brain activity (left: $\Delta R^{2}=0.33, p=$ 0.000006; right: $\left.\Delta R^{2}=0.33, p=0.000004\right)$, demonstrating that the observed relationships between the amygdalae and performance could not be explained by a nonspecific relationship between whole-brain activity and performance. In addition, we also found that the unique contribution of the amygdala to RT was significant while controlling for other regions also correlated with task performance (left: $\Delta R^{2}=0.08, p=0.008$; right: $\left.\Delta R^{2}=0.08, p=0.009\right)$. This result indicates that the amygdala alone has a unique contribution to the prediction of RT above and beyond the contributions of other specific regions found to correlate with RT.

Follow-up ROI analyses. The first ROI analysis tested whether the amygdala-RT relationship generalized beyond stimuli displaying human faces to neutral words. We tested the correlations between amygdala and performance, separately for each category of stimulus contents (words or faces). The correlations between amygdala and RT were significant for both words (left amygdala, $r=-0.40, p=0.003$; right, $r=-0.43, p=0.001$ ) and faces (left amygdala, $r=-0.35, p=0.009$; right, $r=-0.33$, $p=0.016)$. In addition, the magnitude of the correlations did not differ significantly ( $Z P F$ values $<1.65$, for both left and right amygdalae), indicating that the relationship between amygdala and response speed was not differentially affected by stimulus type.

The second ROI analysis used the same methods as the first, but focused instead on whether the amygdala-RT relationship generalized beyond conditions in which emotional moods had been induced. The relationship between the amygdala and RT was observed in the neutral mood condition (left amygdala: $r=$ $-0.43, p=0.001$; right: $r=-0.34, p=0.02$ ), as well as in the emotional conditions separately, positive mood (left amygdala: $r=-0.25, p=0.06$; right: $r=-0.25, p=0.06)$ and negative mood (RTs: left amygdala: $r=-0.30, p=0.03$; right: $r=-0.29$, $p=0.04)$. Comparisons of correlation coefficients across mood conditions were nonsignificant (all $Z P F$ values $<1.65$ ), suggesting that the correlations between amygdala and performance were not dependent on the mood manipulation.

In addition, we tested the association between the amygdalae and RT within a critical condition: the condition with neutral mood and word stimuli, thus, without involvement of affectrelated contents or states. The correlation was significant for the left amygdala $(r=-0.45, p=0.001)$, but did not reach the significance threshold for the right amygdala $(r=-0.20, p=$ 0.15 ), suggesting that the left amygdala activity can predict WM performance independently of the presence of an emotional experimental context.

Third, we examined whether the amygdala-performance correlations could be explained by emotional feelings related to the experimental mood manipulation and assessed through the selfreport POMS (McNair et al., 1971) scales administered after each block of task trials. Similar analyses were computed to control for the effects of individual differences in stable affective personality dispositions (extraversion, neuroticism, BIS-BAS). Multiple regressions showed that the effects of the amygdala on performance were not altered by statistically controlling for variability in state affect ratings or personality, as shown in supplemental Tables 1 and 2 (available at www.jneurosci.org as supplemental material). Finally, correlations between amygdala activity and Raven's matrices were nonsignificant $(r<0.10)$. Together, these ROI analyses demonstrate that amygdala activity predicts 3-back response speed independently of stimulus contents, induced changes in mood, or individual differences in both affective personality traits and fluid intelligence, using strict a priori anatomical criteria.

\section{Experiment 2}

\section{Behavioral results}

As expected, the median RTs in the 3-back condition were significantly longer than those in the 1-back condition (1-back, $750 \mathrm{~ms}$; 3-back, $934 \mathrm{~ms} ; t_{(20)}=8.3$; $\left.p<0.001\right)$. Further, the 3-back condition resulted in a greater false alarm rate (1-back, $1 \%$ of false alarm errors; 3 -back, $\left.6 \% ; t_{(20)}=7.0 ; p<0.001\right)$ and a diminished $d^{\prime}$ signal detection measure (1-back, 3.86; 3-back, $2.52 ; t_{(20)}=$ $-11.2 ; p<0.001)$.

\section{Imaging results}

To provide a strong test of replication, we conducted an ROI analysis using the same anatomically defined amygdala ROIs from the first study. A significant negative correlation between the amplitude of left amygdala responses and task RT in the 3 -back condition was observed $(r=-0.47$; $p<0.05)$, replicating experiment 1 . Similar to experiment 1 , hierarchical regression analyses showed that this relationship could not be explained by a whole-brain correlation with $\mathrm{RT}\left(\Delta R^{2}=0.22 ; p=0.03\right)$.

Interestingly, the amygdala-RT relationship was specific to the 3 -back condition, because the correlation was opposite in sign in the 1-back condition $(r=0.49 ; p<0.05)$. A comparison of the correlations across conditions confirmed that the relationship between amygdala and RT was significantly moderated by cognitive load $(Z P F=2.71 ; p=0.003)$ (Fig. 4). To further explore this effect, we proceeded to test whether amygdala activity in one condition could predict activity in the other condition. A significant negative correlation was found between 3-back and 1-back amygdala activity $(r=-0.44 ; p=0.05)$, suggesting that the individuals with the highest amygdala activity in the 3-back were also the individuals with the lowest activity in the 1-back condition.

These results suggest that the tendency to have both high amygdala reactivity in a high-load condition and low amygdala activity in a low-demand condition might be a characteristic predicting better performance. To test this hypothesis, we separated the sample in two groups of high (H-P) and low performers (L-P) (see Materials and Methods) and tested a cognitive load (3-back vs 1 -back) by performance (H-P vs L-P) ANOVA. Consistent with the hypothesis, we found an interaction between performance group ( $\mathrm{H}-\mathrm{P}$ vs L-P) and cognitive load $\left(F_{(1,19)}=4.4 ; p=\right.$ $0.05)$. Simple effects showed that the H-P group had significantly higher activity in the 3-back than in the 1-back condition $\left(F_{(1,10)}=4.9, p=0.05\right)$, whereas this difference was nonsignificant for the L-P group $\left(F_{(1,9)}=1.1 ; p=0.31\right)$, as shown in Figure 5. The main effects of cognitive load $(F<1)$ and per- 


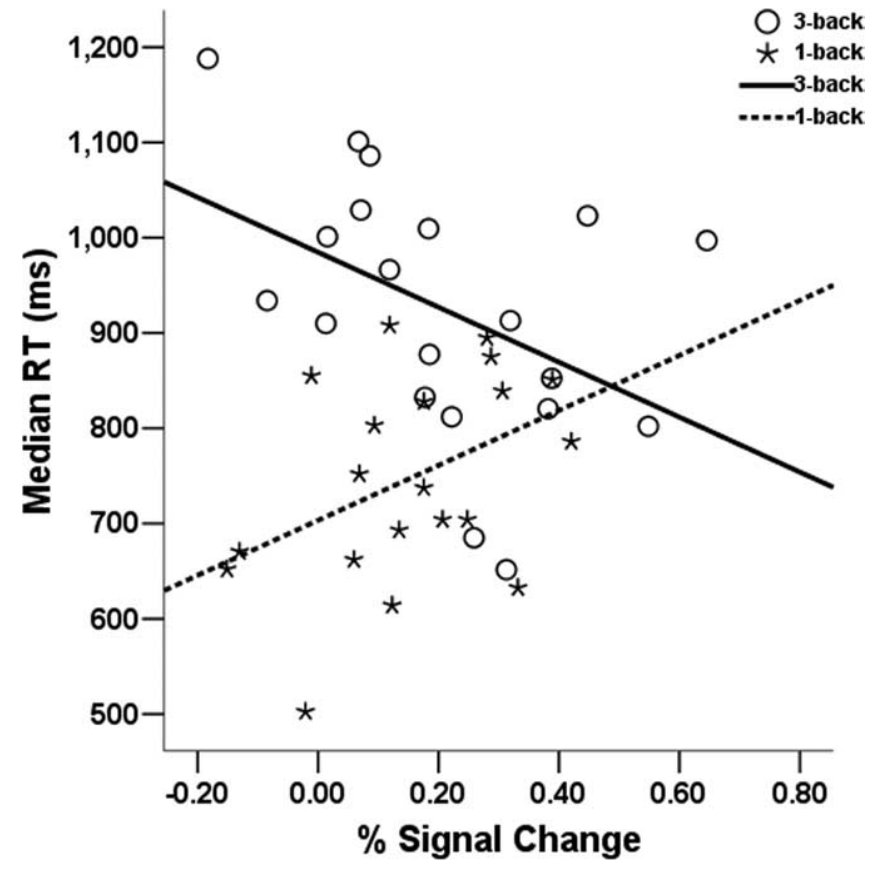

Figure 4. Correlations between anatomical ROl of the left amygdala and task response time by WM load condition for experiment 2 (after controlling for outliers). Correlations are significant for the 3-back condition $(r=-0.47 ; p<0.05)$ and for the 1-back condition $(r=0.49$; $p<0.05$ ).

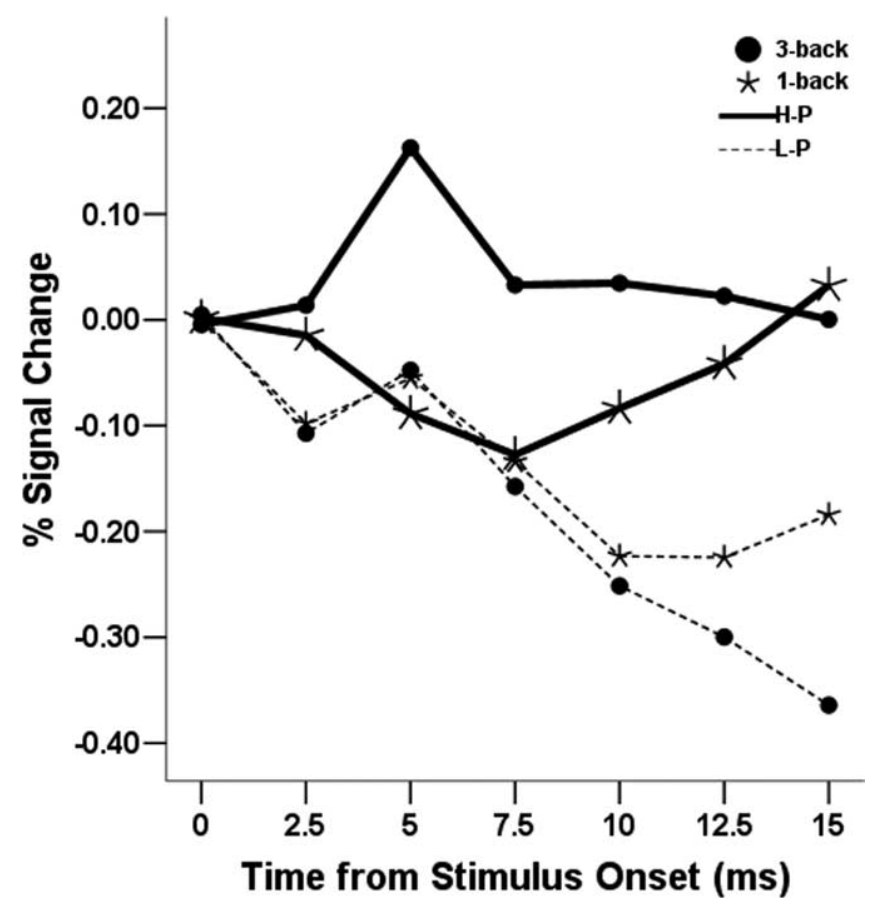

Figure 5. Averaged time course of the left amygdala ROI by WM load condition and performance group for experiment 2 after baseline correction. The effect of cognitive load is specific to the H-P group (see Results).

formance group $\left(F_{(1,19)}=1.3 ; p=0.27\right)$ were not significant. As an additional confirmation of the hypothesis, we found that individual differences in a score of amygdala increase from 1 -back to 3-back conditions strongly predicted RT in both the 3-back $(r=-0.73 ; p<0.001)$ and 1-back conditions $(r=$ $-0.63, p<0.005$ ) (Figure 6).

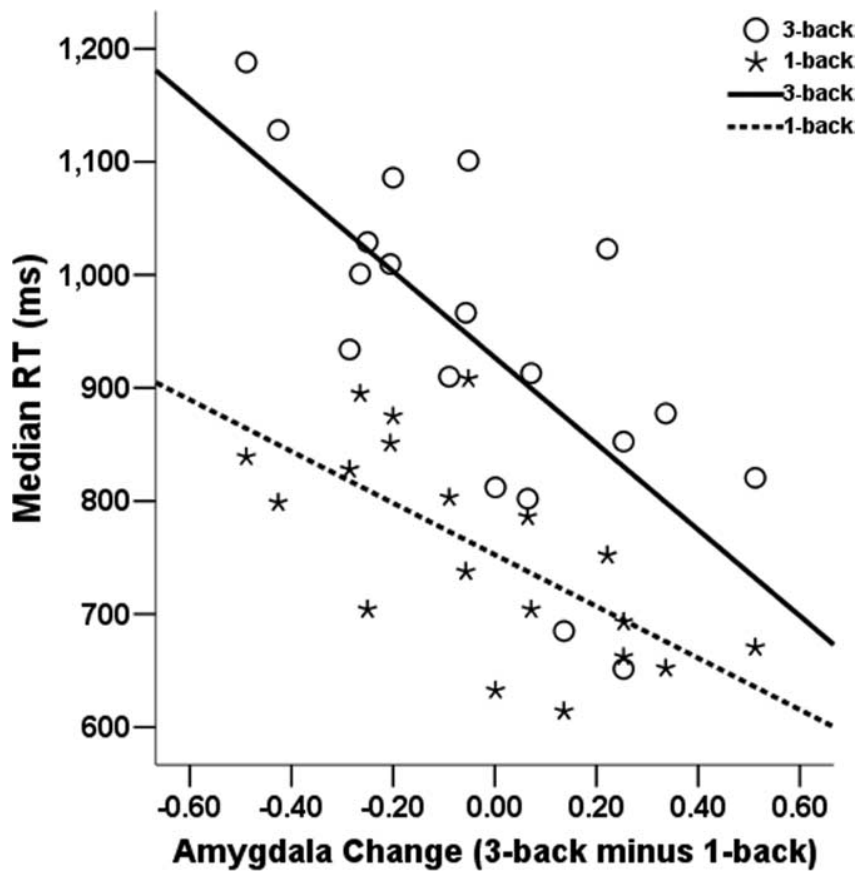

Figure 6. The $x$-axis represents a difference in amygdala activity (3-back minus 1-back) using an anatomical ROI of the left amygdala, and the $y$-axis represents median response time for experiment 2 (after controlling for outliers). Correlations are significant for the 3 -back con$\operatorname{dition}(r=-0.73 ; p<0.001)$ and for the 1-back condition $(r=-0.63 ; p<0.005)$.

Mirroring the neutral mood-only words condition of experiment 1 , the correlation between the right amygdala ROI and RT did not reach the significance threshold but was in the same direction $(r=-0.22)$, suggesting that the left amygdala is a more reliable predictor of cognitive performance than the right amygdala. However, an ANOVA revealed a significant main effect of cognitive load on right amygdala activity $\left(F_{(1,19)}=4.4 ; p=0.05\right)$, and the other effects were not significant $(F<1)$. This result shows that the group-averaged right amygdala activity was significantly higher in the 3-back compared with the 1-back condition, independently of performance group (Fig. 7), indicating that the right amygdala is modulated by cognitive load, but is less sensitive than the left amygdala to interindividual variability in cognitive performance.

In conclusion, we replicated a significant negative correlation between variation in left amygdala activity and RT in a 3-back working memory task involving only neutral words without mood manipulation, using the same anatomical ROIs used in the first study. Further, we found that this relationship was specific to a high WM load. Further, we found evidence suggesting that a tendency to have both high amygdala activity in the 3-back and low amygdala activity in the 1-back condition predicted an improvement of performance in both conditions. Finally, we found evidence suggesting a lateralization of the sensitivity of the amygdala to individual differences in cognitive performance.

\section{Discussion}

In two independent samples, individual differences in eventrelated amygdala activity were significantly related to the speed of task performance in a 3-back WM task. Individuals with stronger amygdala responses (i.e., greater signal change) tended to have the fastest 3-back response times. In addition, we found no evidence that the relationship between amygdala activity and RT was contingent on affect-related variables often associated with 


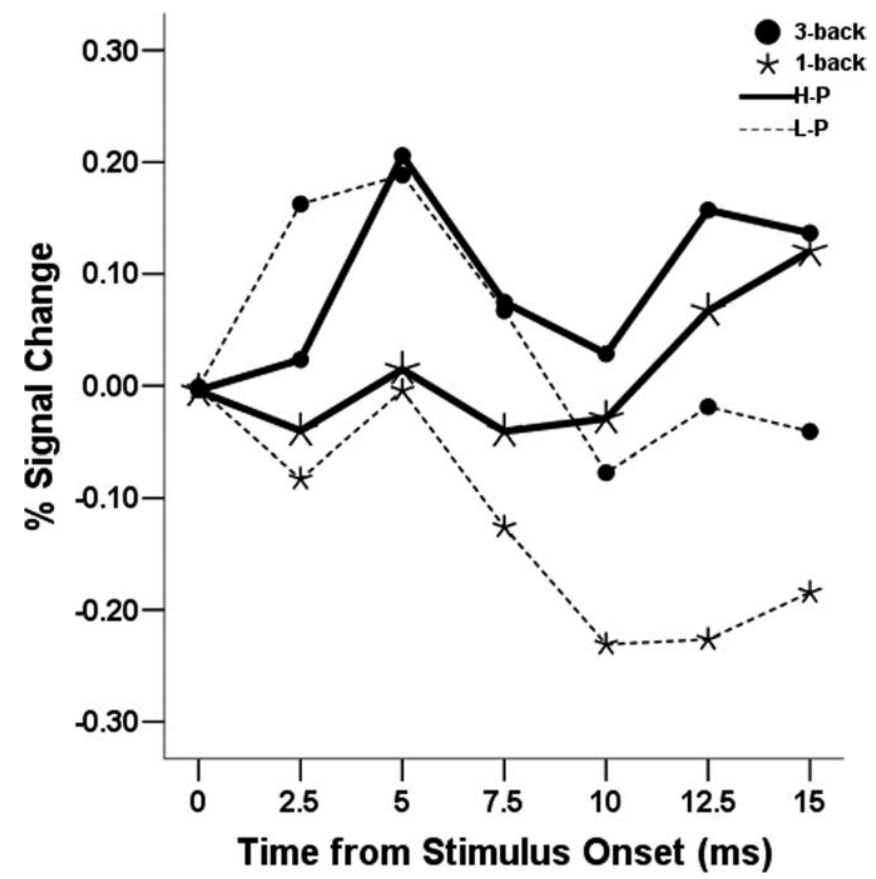

Figure 7. Averaged time course of the right amygdala ROl by WM load condition and performance group for experiment 2. The main effect of cognitive load is significant independently of performance group (see Results).

amygdala activity (face stimuli, emotional states, and personality traits). Conversely, we did find that the correlation between the amygdala and RT varied with respect to a cognitive variable, WM load. Specifically, the amplitude of left amygdala responses was associated with improved performance in the high WM load 3-back condition, whereas it was associated with worse performance in the low WM load 1-back condition.

Our findings are counterintuitive because they show that a brain structure widely thought to be involved primarily in emotion processing is also involved in a WM task independently of affect-related variables. Further, the effect sizes and the replication suggest that these results are robust and generalize to the population of healthy adults from which the sample of participants was drawn. These findings do not challenge the notion of the involvement of the amygdala in emotional processing. They argue instead for the need of a comprehensive account of amygdala function that integrates the well established sensitivity of the amygdala to emotional stimuli with the current and other extant data showing its facilitating role in higher cognition.

To speculate, one possible explanation is based on the fact that the 3-back task is a challenging, difficult task because it is demanding in processing resources, as are most tasks recruiting executive control. We hypothesize, as have others, that the amygdala may serve a general vigilance function (Davis and Whalen, 2001) aimed at preparing the organism to better cope with such challenging situations. The vigilance response of the amygdala could take the form of phasic neuromodulatory signals projected to cognitive, motor, and/or autonomic nervous systems during a situation demanding an unusual addition of metabolic resources, such as a situation of physical threat or a complex mental task. More particularly, signals sent to neocortical areas might enhance attentional processing of environmental stimuli, which has an obvious adaptive value during challenging situations. This attentional enhancement might be either nonspecific, facilitating the processing of all incoming stimuli, or selective, facilitating only goal-relevant stimuli (i.e., stimuli carrying information important to resolve the current challenge). It is noteworthy that goal processing is a fundamental component of both emotion (Frijda, 1986; Scherer, 2001) and executive control (Anderson, 1983; Braver and Cohen, 2000). The vigilance response to motivationally relevant information may be the core basis of most emotional and stress responses, preparing the organism to cope with challenging situations. Accordingly, it can be speculated that the difficulty of 3-back trials in our experiments triggered subtle "stress" responses to task stimuli leading to a modulation of attentional functions by the amygdala.

This explanation is consistent with results suggesting that the amygdala modulates brain systems linked to higher cognition. For instance, several studies indicate that the amygdala has anatomical and neurochemical properties making it particularly suitable to modulate higher cognitive functions (Emery and Amaral, 2000; Davis and Whalen, 2001; Ghashghaei and Barbas, 2002). In addition, numerous studies have found evidence that the amygdala modulates cognition in the case of the emotional enhancement of long-term memory (Hamann et al., 1997, 1999; Philippot and Schaefer, 2001; Smith et al., 2005, 2006; LaBar and Cabeza, 2006).

The results of experiment 2 also revealed that amygdala activity was not only related to faster RT in the 3-back condition but also to slower RT in the 1-back condition. Additional analyses indicated that individuals whose amygdala activity was both augmented in the 3-back and dampened in the 1-back condition had a better performance in both conditions. This result tentatively suggests that the amygdala does not have a linear effect on cognition. Instead, the modulation of cognitive systems by the amygdala may be beneficial for challenging situations, but detrimental to low-demand tasks. This interpretation is consistent with findings indicating that the effects of neurotransmitters associated with the amygdala (dopamine and noradrenaline) on cognition are not linear (Barch, 2004). It is also consistent with the classical idea that the optimal level of biological energy necessary to cognitive performance depends on the specific demands of the task (Humphreys and Revelle, 1984).

In addition to the amygdala, our results also revealed other brain structures associated with 3-back behavioral performance, detailed in Table 1. This network of regions is somewhat different from that observed in a previous study of brain-behavior relationships in WM (Pessoa et al., 2002b), that identified performance correlations in regions such as the inferior frontal gyrus, frontal eye fields, and parietal regions. The difference with our results is probably caused by multiple factors, such as the absence of a strong "updating" component and the stronger demand on fine-grained perceptual processing in the task used by Pessoa et al (2002b). In addition, Pessoa et al. (2002b) adopted a withinsubjects approach to brain-behavior relationships, which can potentially lead to different results than a between-subjects approach (Braver and Ruge, 2006). Nonetheless, there are also clear convergences between Pessoa et al. (2002b) and our results, such as relationships between performance and both the insula and the Brodmann's area (BA)6/BA8 area. Specifically, the convergence of a BA6 activation across both studies may reflect a common WM storage component, which is consistent with previous studies (Smith and Jonides, 1999; Lau et al., 2004; Owen et al., 2005; Braver and Ruge, 2006).

An alternative view of our results stems from previous studies showing a signal decrease in the amygdala during tasks requiring cognitive effort (Shulman et al., 1997; Gusnard et al., 2001). According to this view, the correlation between performance and 
left amygdala activity in the 3-back task might be the result of an attenuated decrease of amygdala activity in high-performers, because the task would be easier for them. Because experiment 2 included an easy low-load condition (1-back), it enabled us to test a critical prediction deriving from this alternative explanation: compared with the easy (1-back) condition, amygdala activity in the difficult (3-back) condition should significantly decrease for low-performers, and decrease or stay equal (but not increase) for high-performers. In the left amygdala, we found no significant difference in amygdala activity for low performers, and a significant increase of amygdala activity in the 3-back compared with the 1-back condition in high performers, which makes the alternative explanation unlikely. Higher activity in the 3-back compared with the 1-back condition was also found in the right amygdala, which further contradicts the view that 3-back activity in the amygdala would be a decrease relative to an easier condition. This apparent contradiction with the findings mentioned above is probably caused by the fact that they used block-related activity, whereas we used event-related activity. To address this issue, we reanalyzed our data using block-related activity. Using an ROI analysis, we found a significant decrease in blocked left amygdala activity in the 3-back compared with the 1-back condition $(t=3.0 ; p=0.007)$, thus consistent with previous research using block-related activity. This is consistent with previous findings suggesting that phasic and temporally sustained brain activity can reflect different functional properties (Donaldson et al., 2001; Braver et al., 2003).

Together, our results point to several questions for future research. First, our results argue for a more comprehensive understanding of the functions of the human amygdala. In particular, the amygdala may subserve motivational functions that are deeper, but common to both emotion and higher cognition. Vigilance and the detection of goal-relevance seem to be serious candidates. Second, future research might determine whether different subdivisions of the amygdala (e.g., dorsal and ventral amygdalae) (Davis and Whalen, 2001) play specific roles in the modulation of higher cognition. Third, our results suggest that both left and right amygdalae are affected by a within-subjects manipulation of cognitive load, but the left amygdala may be a more sensitive predictor of interindividual differences in WM performance. These findings argue for the need of additional research to better understand the role of lateral differences of the amygdala in higher cognition.

In summary, our findings show that individuals who have high levels of amygdala BOLD signal during a difficult working memory task are also the ones who perform better, across two different samples of human subjects. This effect was independent of task content, as well as of any overt emotional factor, whereas it was significantly moderated by cognitive load: the relationship between higher amygdala activity and faster RT was specific to a condition with high cognitive load. Further, they show that the amplitude of amygdala responses is higher in a high-load condition than a low-load WM condition. These results argue for a more comprehensive account of the functions of the human amygdala, integrating data from its involvement in both emotion and higher cognition.

\section{References}

Aalto S, Bruck A, Laine M, Nagren K, Rinne JO (2005) Frontal and temporal dopamine release during working memory and attention tasks in healthy humans: a positron emission tomography study using the high-affinity dopamine $\mathrm{D}_{2}$ receptor ligand [11C]FLB 457. J Neurosci 25:2471-2477.

Anderson JR (1983) The architecture of cognition. Cambridge, MA: Harvard UP.
Barch DM (2004) Pharmacological manipulation of human working memory. Psychopharmacology (Berl) 174:126-135.

Braver TS, Cohen JD (2000) On the control of control: the role of dopamine in regulating prefrontal function and working memory. In: Attention and performance XVIII (Monsell S, Driver J, eds), pp 713-738. Cambridge, MA: MIT.

Braver TS, Ruge H (2006) Functional neuroimaging of executive functions. In: Functional neuroimaging of cognition (Cabeza R, Kingstone A, eds), pp 307-347. Cambridge, MA: MIT.

Braver TS, Reynolds JR, Donaldson DI (2003) Neural mechanisms of transient and sustained cognitive control during task switching. Neuron 39:713-726.

Carver CS, White T (1994) Behavioral inhibition, behavioral activation, and affective responses to impending reward and punishment: The BIS/BAS scales. J Pers Soc Psychol 67:319-333.

Cohen J, MacWhinney B, Flatt M, Provost J (1993) PsyScope: an interactive graphic system for designing and controlling experiments in the psychology laboratory using Macintosh computers. Behav Res Methods Instrum Comput 25:257-271.

Damasio AR, Grabowski TJ, Bechara A, Damasio H, Ponto LL, Parvizi J, Hichwa RD (2000) Subcortical and cortical brain activity during the feeling of self-generated emotions. Nat Neurosci 3:1049-1056.

Davis M, Whalen PJ (2001) The amygdala: vigilance and emotion. Mol Psychiatry 6:13-34.

Donaldson DI, Petersen SE, Ollinger JM, Buckner RL (2001) Dissociating item and state components of recognition memory using fMRI. NeuroImage 13:129-142.

Emery NJ, Amaral DG (2000) The role of amygdala in primate social cognition. In: Cognitive neuroscience of emotion (Lane RD, Nadel L, eds), pp 156-191. New York: Oxford UP.

Eysenck HJ, Eysenck SBG (1975) Manual of the eysenck questionnaire. London: Hodder and Stoughton.

Fried I, Wilson CL, Morrow JW, Cameron KA, Behnke ED, Ackerson LC, Maidment NT (2001) Increased dopamine release in the human amygdala during performance of cognitive tasks. Nat Neurosci 4:201-206.

Frijda NH (1986) The emotions. London: Cambridge UP.

Friston KJ, Holmes AP, Worsley KJ, Poline JP, Frith CD, Frackowiak RSJ (1995) Statistical parametric mapping in functional imaging: a general linear approach. Hum Brain Mapp 2:189-210.

Ghashghaei HT, Barbas H (2002) Pathways for emotion: interactions of prefrontal and anterior temporal pathways in the amygdala of the rhesus monkey. Neuroscience 115:1261-1279.

Gray JR (2001) Emotional modulation of cognitive control: Approachwithdrawal states double-dissociate spatial from verbal two-back task performance. J Exp Psychol Gen 130:436-452.

Gray JR, Braver TS, Raichle ME (2002) Integration of emotion and cognition in the lateral prefrontal cortex. Proc Natl Acad Sci USA 99:4115-4120.

Gray JR, Chabris CF, Braver TS (2003) Neural mechanisms of general fluid intelligence. Nat Neurosci 6:316-322.

Gray JR, Burgess GC, Schaefer A, Yarkoni T, Larsen RJ, Braver TS (2005) Personality differences in neural processing efficiency revealed using fMRI. Cogn Affect Behav Neurosci 5:182-190.

Gusnard DA, Akbudak E, Shulman GL, Raichle ME (2001) Medial prefrontal cortex and self-referential mental activity: relation to a default mode of brain function. Proc Natl Acad Sci USA 98:4259-4264.

Hamann SB, Cahill L, McGaugh JL, Squire LR (1997) Intact enhancement of declarative memory for emotional material in amnesia. Learn Mem 4:301-309.

Hamann SB, Ely TD, Grafton ST, Kilts CD (1999) Amygdala activity related to enhanced memory for pleasant and aversive stimuli. Nat Neurosci 2:289-293.

Holland PC, Gallagher M (1999) Amygdala circuitry in attentional and representational processes. Trends Cogn Sci 3:65-73.

Holland PC, Gallagher M (2006) Different roles for amygdala central nucleus and substantia innominata in the surprise-induced enhancement of learning. J Neurosci 26:3791-3797.

Humphreys MS, Revelle W (1984) Personality, motivation, and performance: A theory of the relationship between individual differences and information processing. Psychol Rev 91:153-184.

Kapp BS, Whalen PJ, Supple W, Pascoe JP (1992) Amygdaloid contributions to conditioned arousal and sensory information processing. In: The 
amygdala: neurobiological aspects of emotion, memory and mental dysfunction. (Aggleton JP, ed), pp 229-254. New York: Wiley.

Kosslyn SM, Cacioppo JT, Davidson RJ, Hugdahl K, Lovallo WR, Spiegel D, Rose R (2002) Bridging psychology and biology: The analysis of individuals in groups. Am Psychol 57:341-351.

LaBar KS, Cabeza R (2006) Cognitive neuroscience of emotional memory. Nat Rev Neurosci 7:54-64.

Lau HC, Rogers RD, Ramnani N, Passingham RE (2004) Willed action and attention to the selection of action. NeuroImage 21:1407-1415.

LeDoux JE (1996) The emotional brain: the mysterious underpinnings of emotional life. New York: Simon and Schuster.

McAvoy MP, Ollinger JM, Buckner RL (2001) Cluster size thresholds for assessment of significant activation in fMRI. NeuroImage 13:S198.

McIntyre CK, Marriott LK, Gold PE (2003) Cooperation between memory systems: acetylcholine release in the amygdala correlates positively with performance on a hippocampus-dependent task. Behav Neurosci 117:320-326.

McNair DM, Lorr M, Droppleman LF (1971) EITS Manual for the Profile of Mood States. San Diego: Educational and Industrial Testing Service.

Morris JS, Frith CD, Perrett DI, Rowland D, Young AW, Calder AJ, Dolan RJ (1996) A differential neural response in the human amygdala to fearful and happy facial expressions. Nature 383:812-815.

Owen AM, McMillan KM, Laird AR, Bullmore E (2005) N-back working memory paradigm: a meta-analysis of normative functional neuroimaging studies. Hum Brain Mapp 25:46-59.

Peinado-Manzano MA (1990) The role of the amygdala and the hippocampus in working memory for spatial and non-spatial information. Behav Brain Res 38:117-134.

Pessoa L, McKenna M, Gutierrez E, Ungerleider LG (2002a) Neural processing of emotional faces requires attention. Proc Natl Acad Sci USA 99:11458-11463.

Pessoa L, Gutierrez E, Bandettini P, Ungerleider L (2002b) Neural correlates of visual working memory: fMRI amplitude predicts task performance. Neuron 35:975-987.

Phelps EA (2005) The interaction of emotion and cognition: insights from studies of the human amygdala. In: Emotion and consciousness (Feldman-Barrett L, Niedenthal PM, Winkielman P, eds), pp 51-66. New York: Guilford.

Philippot P, Schaefer A (2001) Emotion and memory. In: Emotion: current issues and future directions (Mayne TJ, Bonanno GA, eds), pp 82-122. New York: Guilford.
Raghunathan TE, Rosenthal R, Rubin DR (1996) Comparing correlated but nonoverlapping correlations. Psychol Methods 1:178-183.

Raven J, Raven JC, Court JH (1998) Manual for Raven's Progressive Matrices and Vocabulary Scales. Oxford: Oxford Psychologists.

Sander D, Grafman J, Zalla T (2003) The human amygdala: an evolved system for relevance detection. Rev Neurosci 14:303-316.

Scherer KR (2001) Appraisal considered as a process of multilevel sequential checking. In: Appraisal processes in emotion: theory, methods, research (Scherer KR, Schorr A, Johnstone T, eds), pp 92-120. New York: Oxford UP.

Shulman GL, Fiez JA, Corbetta M, Buckner RL, Miezin FM, Raichle ME, Petersen SE (1997) Common blood flow changes across visual tasks: II Decreases in cerebral cortex. J Cogn Neurosci 9:648-663.

Smith AP, Henson RN, Rugg MD, Dolan RJ (2005) Modulation of retrieval processing reflects accuracy of emotional source memory. Learn Mem 12:472-479.

Smith AP, Stephan KE, Rugg MD, Dolan RJ (2006) Task and content modulate amygdala-hippocampal connectivity in emotional retrieval. Neuron 49:631-638.

Smith EE, Jonides J (1999) Storage and executive processes in the frontal lobes. Science 283:1657-1661.

Snodgrass JG, Corwin J (1988) Pragmatics of measuring recognition memory: applications to dementia and amnesia. J Exp Psychol Gen 117:34-50

Stern RM, Ray WJ, Quigley KS (2001) Psychophysiological recording. New York: Oxford UP.

Talairach J, Tournoux P (1988) Co-planar stereotaxic atlas of the human brain 3-dimensional proportional system: an approach to cerebral imaging. Stuttgart: Thieme.

Visscher KM, Miezin FM, Kelly JE, Buckner RL, Donaldson DI, McAvoy MP, Bhalodia VM, Petersen SE (2003) Mixed blocked/event-related designs separate transient and sustained activity in fMRI. NeuroImage 19:1694-1708

Vuilleumier P, Armony JL, Driver J, Dolan RJ (2001) Effects of attention and emotion on face processing in the human brain: an event-related fMRI study. Neuron 30:829-841.

Whalen PJ (1998) Fear, vigilance and ambiguity: initial neuroimaging studies of the human amygdala. Curr Dir Psychol Sci 7:177-188.

Whalen PJ, Kagan J, Cook RG, Davis FC, Kim H, Polis S, McLaren DG, Somerville LH, McLean AA, Maxwell JS, Johnstone T (2004) Human amygdala responsivity to masked fearful eye whites. Science 306:2061.

Zald DH (2003) The human amygdala and the emotional evaluation of sensory stimuli. Brain Res Brain Res Rev 41:88-123. 